Published in final edited form as:

Cancer. 2015 November 15; 121(22): 3990-3997. doi:10.1002/cncr.29631.

\title{
Disparities in early death and survival in children, adolescents and young adults with acute promyelocytic leukemia in California
}

\author{
Renata Abrahão, MD, MSc ${ }^{1,2}$, Raul C. Ribeiro, MD ${ }^{3}$, Bruno C. Medeiros, MD ${ }^{4}$, Ruth H. \\ Keogh, DPhil ${ }^{5}$, and Theresa H.M. Keegan, PhD, MSc ${ }^{2,6}$ \\ ${ }^{1}$ Department of Non-Communicable Disease Epidemiology, London School of Hygiene and \\ Tropical Medicine, London, GB \\ ${ }^{2}$ Cancer Prevention Institute of California, Fremont, CA \\ ${ }^{3}$ Department of Oncology, Leukemia and Lymphoma Division, St. Jude Children's Research \\ Hospital, Memphis, TN \\ ${ }^{4}$ Division of Hematology, Stanford University School of Medicine, Stanford, CA \\ ${ }^{5}$ Department of Medical Statistics, London School of Hygiene and Tropical Medicine, London, GB \\ ${ }^{6}$ Division of Epidemiology, Department of Health Research and Policy, Stanford, CA
}

\section{Abstract}

Background-Findings from clinical trials and population-based studies have differed as to whether mortality within 30 days of diagnosis (early death) of acute promyelocytic leukemia has decreased in the era of all-trans retinoic acid (ATRA) and anthracycline-based chemotherapy.

Methods-We investigated 7- and 30-day mortality and survival in 772 patients aged 0-39 years when diagnosed with APL during 1988-2011, using data from the California Cancer Registry. We used logistic regression and Cox proportional models to examine the association of early death and survival, respectively, with sociodemographic and clinical factors.

Results-Overall 30-day mortality decreased significantly over time, from $26 \%$ (1988-1995) to 14\% (2004-2011) ( $P=0.004)$. In multivariable analysis, the odds of 30-day mortality were 3 times as high during 1988-1995 than 2004-2011 ( $P=0.001)$. However, 7-day mortality did not improve over time $(P=0.229)$. When patients who died within 7 days of diagnosis were excluded, 30-day mortality during 1996-2011 was 3\%-8\%, similar to levels reported in clinical trials. Higher early

Correspondence: Renata Abrahão, Cancer Prevention Institute of California, 2201 Walnut Avenue, Suite 300, Fremont, CA, 94538 , renataabrahao8901@gmail.com or renata.abrahao@1shtm.ac.uk, Phone: (415) 623-9944; Fax: (510) 608-5095.

Conflict of interest: We declare no competing interests.

Authorship contributions

RA performed and RHK advised on the statistical analyses, RCR, RHK and BCM interpreted the data and drafted and reviewed the manuscript. RA and THMK designed the study, interpreted the data, and led the writing and review of the manuscript. All authors read and approved the final manuscript.

Institutional Review Board (IRB) approval - Ethics approval for human subjects research was obtained from the Cancer Prevention Institute of California Institutional Review Board. As the analysis was based on state-mandated cancer registry data, the study was conducted in accordance with the waivers of individual informed consent and HIPAA authorization. 
death and lower survival were associated with lack of health insurance (1996-2011) (early death $\mathrm{OR}=2.67, P=0.031$ ) and Hispanic race/ethnicity (early death $\mathrm{OR}=2.13, P=0.014$ ). Early death was not associated with age, sex, socioeconomic status or hospital type. Black patients also experienced worse survival.

Conclusions-Our findings revealed a decreased 30-day mortality during the ATRA era, but 7day mortality remained high. Efforts to achieve equal outcomes in young patients with APL should focus on improving access to effective treatment, mainly among uninsured patients and those of Hispanic and Black race/ethnicity.

\section{Keywords}

acute promyelocytic leukemia; ATRA; early death; survival; children; adolescents; young adults; health insurance; health disparities

\section{INTRODUCTION}

Acute promyelocytic leukemia (APL) is a subtype of acute myeloid leukemia (AML) that carries the PML/RAR-a fusion in more than $90 \%$ of cases. Bleeding and thrombosis are frequent and can be aggravated by cytotoxic chemotherapy, resulting in early death due mainly to intracranial hemorrhage. ${ }^{1}$

An estimated $600-800$ new cases of APL (4\%-13\% of AML cases) occur annually in the U.S., most frequently in adults. ${ }^{2,3}$ While APL was once highly fatal, the addition of alltrans retinoic acid (ATRA) to anthracycline-based chemotherapy and the introduction of arsenic trioxide (arsenic) have dramatically improved outcomes; currently, 95\%-100\% of patients with APL gain complete remission. ${ }^{4,5}$ Moreover, arsenic has become the treatment of choice for relapsed APL after frontline treatment with ATRA and chemotherapy. ${ }^{6}$

ATRA and arsenic rapidly reduce the risk of hemorrhage and should be initiated as soon as APL is suspected. ${ }^{7}$ ATRA was approved by the U.S. Food and Drug Administration (FDA) in November, 1995 and arsenic in September, 2000. During the ATRA era, early death has decreased overall, from approximately $20 \%{ }^{8,9}$ to $5 \%-10 \% .{ }^{10}$ However, early death remains high in the U.S. ${ }^{11,12}$ and Europe, ${ }^{13}$ implicating factors other than ATRA.

Because recent studies have examined early death and survival in patients aged $\geq 15$ years ${ }^{11,13,14}$ and there are few reports of population-based studies in young patients with APL (Supplementary Table S1), we investigated early death and survival in patients in California, diagnosed at ages 0-39 years over a 25-year period, and assessed the association of sociodemographic and clinical factors with these outcomes.

\section{PATIENTS AND METHODS}

\section{Patient selection}

Data were obtained from the California Cancer Registry, to which reporting is mandatory and completeness of cases is at least $98 \% .{ }^{15}$ We identified all patients with a first, primary APL diagnosed at age 0 to 39 years during 1988-2011 and followed until December 31, 
2012. APL was diagnosed as histology code 9866 in the International Classification of Diseases for Oncology, third edition. ${ }^{16}$ Of 784 patients identified, 4 were excluded due to missing date of diagnosis and 8 due to unknown or Native American (small subgroup) race/ ethnicity. Our study included 772 patients.

\section{Variables}

The variables examined for association with APL outcomes were age at diagnosis, categorized as four groups based on progressive decrements in survival ${ }^{17}(0-9,10-19,20-$ 29 and 30-39 years); sex; era of diagnosis according to ATRA approval by the U.S. FDA (pre-ATRA era, 1988-1995; earlier ATRA era, 1996-2003; and later ATRA era, 20042011); race/ethnicity (non-Hispanic white [white], non-Hispanic black [black], Hispanic, and Non-Hispanic Asian/Pacific Islander [Asian]); initial care at hospitals affiliated with National Cancer Institute (NCI)-designated cancer centers; type of health insurance at admission (routinely documented starting in 1996) (none, public, private or unknown/not otherwise specified); and neighborhood socioeconomic status (SES) based on block-level census data. Neighborhood SES quintiles based on statewide distribution have been utilized extensively in California. ${ }^{18}$

Information on hospital designation was from the initial reporting facility. There were no data on intensity of treatment or drugs used (conventional genotoxic chemotherapy, ATRA and/or arsenic).

\section{Statistical analysis}

We used univariable and multivariable logistic regression to investigate the association of the sociodemographic and clinical factors with 7- and 30-day mortality, through estimation of the odds ratios (ORs) and associated 95\% confidence intervals (CIs). We analyzed 30-day mortality with and without patients who died within 7 days. We estimated overall survival (all-cause survival) at 1 and 5 years by using the Kaplan-Meier method, and compared differences in survival across strata for each variable using the log-rank test. We used univariable and multivariable Cox regression models to examine the association of sociodemographic and clinical factors with the risk of death, through estimation of the hazard ratios (HRs) and associated 95\% CIs. Schoenfeld residuals were used to assess the proportional hazard assumptions. We tested for interactions between calendar periods, age groups, neighborhood SES and race/ethnicity. All statistical analyses we performed by using the Stata 13 software. A two-sided $P<0.05$ was considered statistically significant.

\section{RESULTS}

Approximately $16 \%$ of all AML cases in the registry were APL, most of which (79\%) were diagnosed during the ATRA era (after 1995). According to death certificates, most patients died of leukemia $(n=228,90 \%)$; a much smaller percentage of patients died of other $(n=17$, $7 \%)$ or unknown $(\mathrm{n}=7,3 \%)$ causes. Fewer than $2 \%$ of patients died of complications of APL treatment, such as infection $(n=2)$, renal dysfunction $(n=1)$ or heart failure $(n=1)$. Table 1 summarizes patient characteristics. 


\section{Early death}

Among patients who experienced early death, median age at diagnosis was 29 years; 82 of these patients (11\%) died within 7 days and 133 (17\%) died within 30 days of diagnosis. Thirty-day mortality decreased significantly over the 3 eras from 26\% in 1988-1995 (preATRA) to $16 \%$ in 1996-2003 (earlier ATRA era) to 14\% in 2004-2011 (later ATRA era) $(P=0.004$, Table 1) (Figure 1). However, 7-day mortality showed no evidence of a significant decrease. In a multivariable analysis (Table 2), the odds of 30-day mortality differed significantly between $1988-1995$ and later eras $(P=0.001)$, but not between the 1996-2003 and 2004-2011 eras. Hispanic patients had a risk of 30-day mortality approximately twice that of white patients. After 1995, type of health insurance was significantly associated with both 7-day and 30-day mortality; the risk of 30-day mortality was approximately 3 times as high in uninsured as in privately insured patients [OR=2.67 (95\% CI: 1.10-6.52)]. Early death was not found to differ significantly between patients with private vs. public insurance $(P=0.243)$.

When patients with 7-day mortality $(n=82)$ were excluded from analysis, 30-day mortality decreased from 15\% during 1988-1995 to 8\% during 1996-2003 and 3\% during 2004-2011 $(P<0.0001$; data not shown). There was no evidence of interactions between any variables.

\section{Survival}

During 0-25 years of follow-up (median in entire cohort, 4.4 years), $33 \%$ of patients $(n=252)$ died. Five-year survival increased from 46.7\% during 1988-1995 to $70.1 \%$ during 1996-2003 and 77.3\% during 2004-2011 ( $P<0.0001$, Table 1). Based on the log-rank test, a lower survival estimate was significantly associated with earlier period of diagnosis, male sex, older age at diagnosis, and lack of health insurance (Table 1). In univariable analyses, survival was lower in Hispanic and black vs. white patients and uninsured vs. insured patients. In multivariable models, the 1988-1995 era, black/Hispanic race/ethnicity and lack of health insurance remained significantly associated with the hazard of death (Table 3). There was no evidence of a difference in HR between patients with private vs. public insurance $(P=0.999)$. There was no evidence of violation of the Cox proportional hazard assumptions or of interactions between any variables.

When we excluded patients who died within 30 days of diagnosis in 1996-2011, 5-year survival increased from $77.8 \%$ (95\% CI: $70.7 \%-83.3 \%$ ) to $88.8 \%$ (95\% CI: 82.4\%-93.0\%) among patients aged $0-19$ years, and from $72.5 \%$ (95\% CI: $67.8 \%-76.6 \%$ ) to $86.3 \%$ (95\% CI: $81.9 \%-89.7 \%$ ) among patients aged $20-39$ years (data not shown).

\section{DISCUSSION}

In our population-based study spanning 25 years, 30-day mortality decreased significantly after 1995, coinciding with the introduction of ATRA and guidelines recommending aggressive blood product support and intensive infection prophylaxis and treatment for suspected APL. Nevertheless, 30-day mortality remained higher than that observed in nonAPL subtypes of AML, ${ }^{19}$ and 7-day mortality did not improve over time. Our findings suggest that factors other than ATRA contributed to early death; these may include the 
timing of diagnosis or chemotherapy, hospital availability of ATRA/arsenic during the critical 2-3 days after diagnosis, adequate blood products and infection prophylaxis and treatment. A recent study of randomly selected hospitals in the U.S. found that less than half had ATRA, and one of the main barriers to availability was the absence of ATRA on their formularies. ${ }^{20}$

Patients who suffered early death probably lacked early access to effective treatment and/or were too ill when admitted; ten patients in this study died on the day of diagnosis. The FDA's approval of ATRA (and later, arsenic) may not have resulted in the wide or timely availability of these drugs across all California hospitals.

Moreover, despite the great effectiveness of ATRA and arsenic, treatment may cause severe complications that should be recognized and treated promptly, such as differentiation syndrome. Differentiation syndrome occurs in about $2 \%-31 \%$ of patients receiving induction therapy and can mimic other severe complications, such as pulmonary hemorrhage, renal dysfunction and heart failure. ${ }^{21}$ Because of the abrupt presentation and potential gravity of differentiation syndrome, preemptive use of corticosteroids has been proposed. ${ }^{22}$ The syndrome may be promoted by delaying chemotherapy after ATRA, ${ }^{23}$ and delaying ATRA itself for more than 2 days may increase the risk of fatal hemorrhage. ${ }^{24}$ These findings confirm the importance of early diagnosis, rapid intensive treatment and adequate supportive care.

Importantly, we found that uninsured patients had a higher risk of early death and lower survival estimates than those with private and public insurance, suggesting lack of adequate access to care. Our results are consistent with a previous report of worse survival in uninsured vs. insured AYAs. ${ }^{25}$ Wider insurance coverage is likely to provide better outcomes for these patients. Additionally, early death was higher among Hispanic patients, and survival was lower among black and Hispanic patients, than those among white patients. Similar findings have been reported in children with acute lymphoblastic leukemia (ALL), ${ }^{26,27}$ children with AML (excluding APL) ${ }^{28}$ and adults with AML (including APL). ${ }^{14}$ To provide effective and sustainable treatment to patients with APL - a severe but highly curable disease - efforts should also address the social contributors to health inequity, ${ }^{29}$ such as poverty, inadequate access to transportation, and lack of education resources.

In general, population-based studies, ${ }^{11,12}$ such as ours, show a greater proportion of early death than do multi-institutional protocols. The differing findings may reflect the exclusion of patients who died during the first week or were too ill for chemotherapy in prior studies. ${ }^{30}$ In our study, when we excluded deaths within 7 days, we found 30-day mortality during the ATRA era to approximate that in clinical trials. ${ }^{10,31}$ Similarly, when we excluded patients who died within 30 days of diagnosis, 5-year survival was close to that reported in multi-institutional trials in children and AYAs. ${ }^{32,33}$ These observations suggest that selection bias may contribute to the differences in reported survival and early death between most clinical trials and population-based studies. 
Our study had several limitations. Hospital designation was limited to the location of initial care at the reporting facility, so it is possible that some patients diagnosed at one type of facility were subsequently treated at another. However, $92 \%$ of our patients received at least part of their treatment at this hospital, suggesting that our findings were not substantially influenced by this factor. We also lacked data on patients' risk classification at diagnosis, laboratory data, and blood products administered. Although this information would likely have contributed additional important findings, disease outcomes such as early death and survival are of paramount concern. Survival is a measure of the cancer burden and the health system effectiveness and plays a key role in the development of health policies. ${ }^{34}$ Our large California APL cohort allowed us to compare early death and survival across treatment eras and investigate sociodemographic factors associated with outcome. To our knowledge, this is the first population-based study to investigate the association of race/ethnicity with early death and survival in children with APL and to consider the association of outcome with health insurance, hospital type, age, sex, treatment era and neighborhood SES. Further, unlike previous population-based studies, ${ }^{11,}{ }^{12}, 19$ we were able to assess 7 -day mortality.

In conclusion, our findings indicate a true reduction of 30-day mortality among children and AYAs with APL in California, suggesting adherence to modern therapeutic strategies. However, 7-day mortality remained high, suggesting that factors other than ATRA played a role in early death. We identified subgroups of patients vulnerable to early death and reduced survival, including the uninsured and Hispanic patients. Black patients also experienced worse survival. To improve outcomes among young patients with APL, efforts should focus on improving access to effective treatment, mainly among uninsured patients and those of Hispanic and Black race/ethnicity.

\section{Supplementary Material}

Refer to Web version on PubMed Central for supplementary material.

\section{Acknowledgments}

We thank Daphne Y. Lichtensztajn (CPIC) for biostatistical assistance, Shawky Matta (CPIC) for cancer registry expertise, and Sharon Naron (St. Jude) for editing the manuscript.

\footnotetext{
Funding This work was supported by the Children with Cancer UK (RA), Cancer Center Support (CORE) grant P30 CA021765-30 from the National Institutes of Health (NIH) (RCR), and the American Lebanese Syrian Associated Charities (ALSAC) (RCR); by the Stanford Cancer Institute (THMK) and the California Department of Public Health as part of the mandated statewide cancer reporting program (California Health and Safety Code Section 103885), and the Surveillance, Epidemiology and End Results (SEER) Program of the National Cancer Institute (NCI) under contracts N01-PC-35136 awarded to the Cancer Prevention Institute of California, N02-PC15105 awarded to the Public Health Institute, HHSN261201000140C awarded to the Cancer Prevention Institute of California, HHSN261201000035C awarded to the University of Southern California, and HHSN261201000034C awarded to the Public Health Institute; and the Center for Disease Control and Prevention's National Program of Cancer Registries, under agreements U55/CCR921930-02 awarded to the Public Health Institute and U58DP003862-01 awarded to the California Department of Public Health. The ideas and opinions expressed herein are those of the authors, and endorsement by the State of California Department of Public Health, the NCI, and the Centers for Disease Control and Prevention or their contractors and subcontractors is neither intended nor should be inferred.
} 


\section{References}

1. Soignet SL, Frankel SR, Douer D, et al. United States multicenter study of arsenic trioxide in relapsed acute promyelocytic leukemia. J Clin Oncol. 2001; 19(18):3852-60. [PubMed: 11559723]

2. Ribeiro RC, Rego E. Management of APL in developing countries: epidemiology, challenges and opportunities for international collaboration. Hematology Am Soc Hematol Educ Program. 2006:162-8. [PubMed: 17124056]

3. Sanz MA, Grimwade D, Tallman MS, et al. Management of acute promyelocytic leukemia: recommendations from an expert panel on behalf of the European LeukemiaNet. Blood. 2009; 113(9):1875-91. [PubMed: 18812465]

4. Iland HJ, Bradstock K, Supple SG, et al. All-trans-retinoic acid, idarubicin, and IV arsenic trioxide as initial therapy in acute promyelocytic leukemia (APML4). Blood. 2012; 120(8):1570-80. quiz 752. [PubMed: 22715121]

5. Rego EM, Kim HT, Ruiz-Arguelles GJ, et al. Improving acute promyelocytic leukemia (APL) outcome in developing countries through networking, results of the International Consortium on APL. Blood. 2013; 121(11):1935-43. [PubMed: 23319575]

6. Lengfelder E, Lo-Coco F, Ades L, et al. Arsenic trioxide-based therapy of relapsed acute promyelocytic leukemia: registry results from the European LeukemiaNet. Leukemia. 2015

7. Pui, CH. Acute complications Childhood leukemias. Third. Vol. 672. Cambridge, United Kingdon: University Press; 2012. p. 152-215.

8. Rodeghiero F, Avvisati G, Castaman G, Barbui T, Mandelli F. Early deaths and anti-hemorrhagic treatments in acute promyelocytic leukemia. A GIMEMA retrospective study in 268 consecutive patients. Blood. 1990; 75(11):2112-7. [PubMed: 2189506]

9. Cunningham I, Gee TS, Reich LM, Kempin SJ, Naval AN, Clarkson BD. Acute promyelocytic leukemia: treatment results during a decade at Memorial Hospital. Blood. 1989; 73(5):1116-22. [PubMed: 2930837]

10. Di Bona E, Avvisati G, Castaman G, et al. Early haemorrhagic morbidity and mortality during remission induction with or without all-trans retinoic acid in acute promyelocytic leukaemia. $\mathrm{Br} \mathrm{J}$ Haematol. 2000; 108(4):689-95. [PubMed: 10792270]

11. McClellan JS, Kohrt HE, Coutre S, et al. Treatment advances have not improved the early death rate in acute promyelocytic leukemia. Haematologica. 2012; 97(1):133-6. [PubMed: 21993679]

12. Park JH, Qiao B, Panageas KS, et al. Early death rate in acute promyelocytic leukemia remains high despite all-trans retinoic acid. Blood. 2011; 118(5):1248-54. [PubMed: 21653939]

13. Lehmann S, Ravn A, Carlsson L, et al. Continuing high early death rate in acute promyelocytic leukemia: a population-based report from the Swedish Adult Acute Leukemia Registry. Leukemia. 2011; 25(7):1128-34. [PubMed: 21502956]

14. Patel MI, Ma Y, Mitchell BS, Rhoads KF. Age and Genetics: How Do Prognostic Factors at Diagnosis Explain Disparities in Acute Myeloid Leukemia? Am J Clin Oncol. 2013

15. Hayat MJ, Howlader N, Reichman ME, Edwards BK. Cancer statistics, trends, and multiple primary cancer analyses from the Surveillance, Epidemiology, and End Results (SEER) Program. Oncologist. 2007; 12(1):20-37. [PubMed: 17227898]

16. Fritz, A.; Percy, C.; Jack, A., editors. World Health Organization. International Classification of Diseases for Oncology. third. Geneva: World Health Organization; 2000.

17. Chen Y, Kantarjian H, Wang H, Cortes J, Ravandi F. Acute promyelocytic leukemia: a populationbased study on incidence and survival in the United States, 1975-2008. Cancer. 2012; 118(23): 5811-8. [PubMed: 22707337]

18. Yost K, Perkins C, Cohen R, Morris C, Wright W. Socioeconomic status and breast cancer incidence in California for different race/ethnic groups. Cancer Causes Control. 2001; 12(8):70311. [PubMed: 11562110]

19. Percival ME, Tao L, Medeiros BC, Clarke CA. Improvements in the early death rate among 9380 patients with acute myeloid leukemia after initial therapy: A SEER database analysis. Cancer. 2015

20. Mogasala, N.; Perissinotti, A.; Bixby, D. Clinical Availability of All-Trans Retinoic Acid (ATRA) for Patients with Suspected Acute Promyelocytic Leukemia - Why National Guidelines May Not 
be Followed; Poster session presented at 56th ASH Annual Meeting and Exposition; 2014 Dec 6; San Francisco, CA.

21. Rogers JE, Yang D. Differentiation syndrome in patients with acute promyelocytic leukemia. J Oncol Pharm Pract. 2012; 18(1):109-14. [PubMed: 21364078]

22. Sanz MA, Montesinos P. How we prevent and treat differentiation syndrome in patients with acute promyelocytic leukemia. Blood. 2014; 123(18):2777-82. [PubMed: 24627526]

23. Tallman MS, Manji GA. Don't just stand there, do something: strategies for the prevention of early death in acute promyelocytic leukemia: a commentary. Blood Cells Mol Dis. 2011; 46(2):173-4. [PubMed: 21227722]

24. Altman JK, Rademaker A, Cull E, et al. Administration of ATRA to newly diagnosed patients with acute promyelocytic leukemia is delayed contributing to early hemorrhagic death. Leuk Res. 2013; 37(9):1004-9. [PubMed: 23768930]

25. Aizer AA, Falit B, Mendu ML, et al. Cancer-specific outcomes among young adults without health insurance. J Clin Oncol. 2014; 32(19):2025-30. [PubMed: 24888800]

26. Bhatia S, Sather HN, Heerema NA, Trigg ME, Gaynon PS, Robison LL. Racial and ethnic differences in survival of children with acute lymphoblastic leukemia. Blood. 2002; 100(6):195764. [PubMed: 12200352]

27. Abrahao R, Lichtensztajn DY, Ribeiro RC, et al. Racial/ethnic and socioeconomic disparities in survival among children with acute lymphoblastic leukemia in California, 1988-2011: A population-based observational study. Pediatr Blood Cancer. 2015

28. Aplenc R, Alonzo TA, Gerbing RB, et al. Ethnicity and survival in childhood acute myeloid leukemia: a report from the Children's Oncology Group. Blood. 2006; 108(1):74-80. [PubMed: 16537811]

29. Wong WF, LaVeist TA, Sharfstein JM. Achieving health equity by design. JAMA. 2015; 313(14): 1417-8. [PubMed: 25751310]

30. Estey EH, Hutchinson F. Newly diagnosed acute promyelocytic leukemia: arsenic moves front and center. J Clin Oncol. 2011; 29(20):2743-6. [PubMed: 21646610]

31. Yanada M, Matsushita T, Asou N, et al. Severe hemorrhagic complications during remission induction therapy for acute promyelocytic leukemia: incidence, risk factors, and influence on outcome. Eur J Haematol. 2007; 78(3):213-9. [PubMed: 17241371]

32. Testi AM, Biondi A, Lo Coco F, et al. GIMEMA-AIEOPAIDA protocol for the treatment of newly diagnosed acute promyelocytic leukemia (APL) in children. Blood. 2005; 106(2):447-53. [PubMed: 15677559]

33. Gore SD, Gojo I, Sekeres MA, et al. Single cycle of arsenic trioxide-based consolidation chemotherapy spares anthracycline exposure in the primary management of acute promyelocytic leukemia. J Clin Oncol. 2010; 28(6):1047-53. [PubMed: 20085935]

34. Allemani C, Weir HK, Carreira H, et al. Global surveillance of cancer survival 1995-2009: analysis of individual data for 25,676,887 patients from 279 population-based registries in 67 countries (CONCORD-2). Lancet. 2015; 385(9972):977-1010. [PubMed: 25467588] 

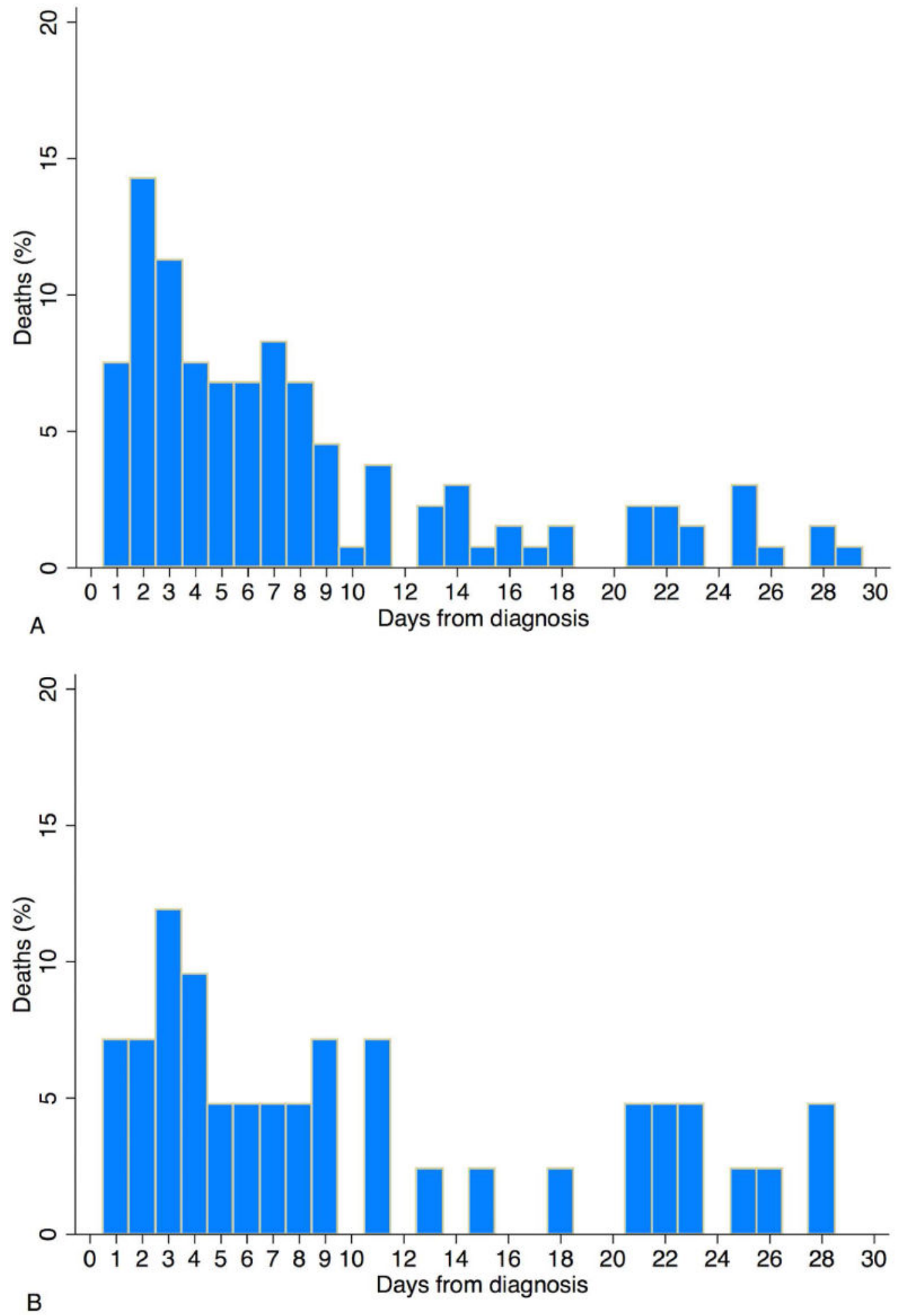

Cancer. Author manuscript; available in PMC 2016 November 15. 

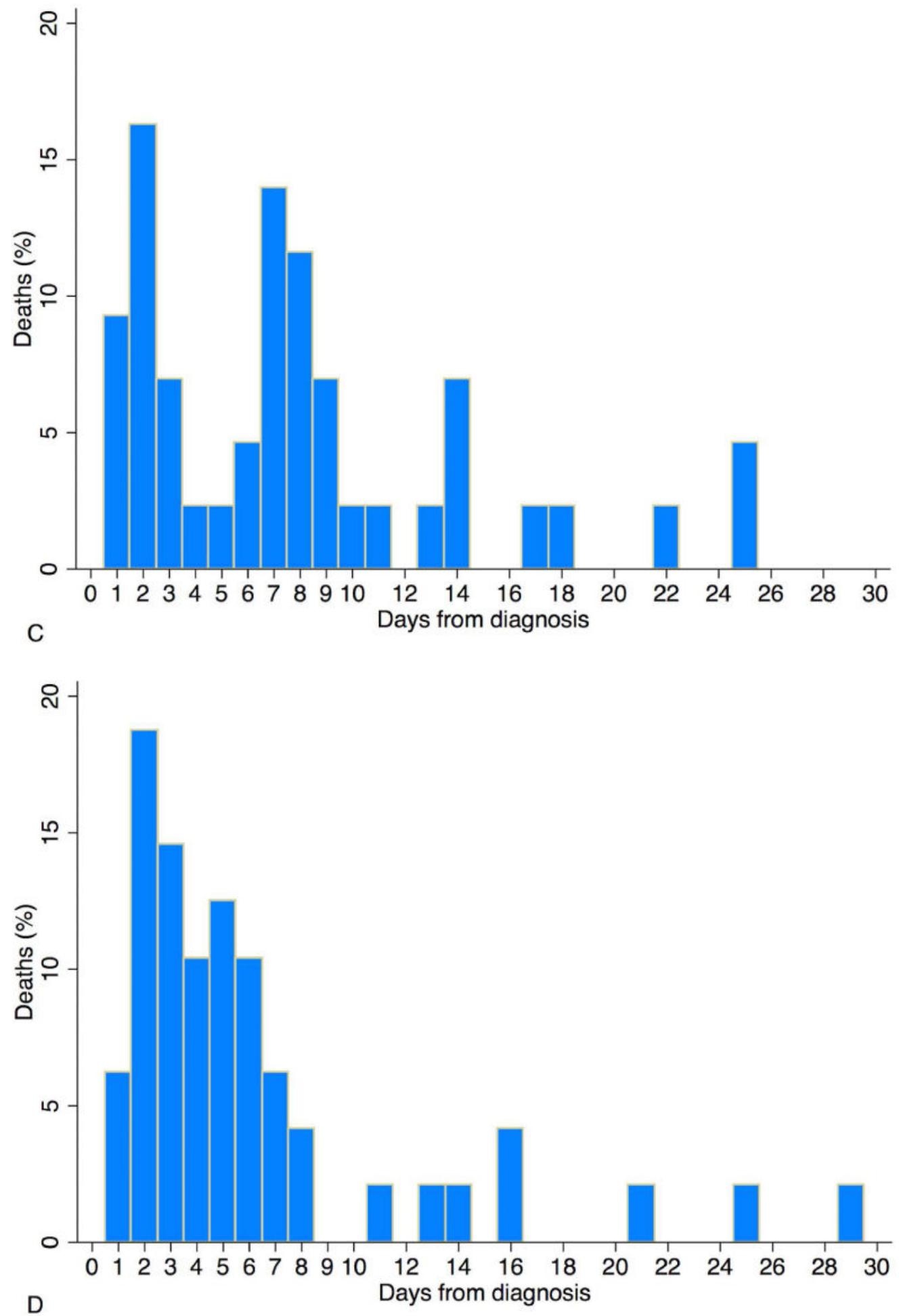

Figure 1. Early death from acute promyelocytic leukemia in California, after diagnosis at age 039 years 
A. Entire study period (1988-2011). B. Pre-ATRA era (1998-1995). C. Earlier ATRA era (1996-2003). D. Later ATRA era (2004-2011). Ten patients who died on the day of diagnosis were considered to have a survival time of 1 day. 


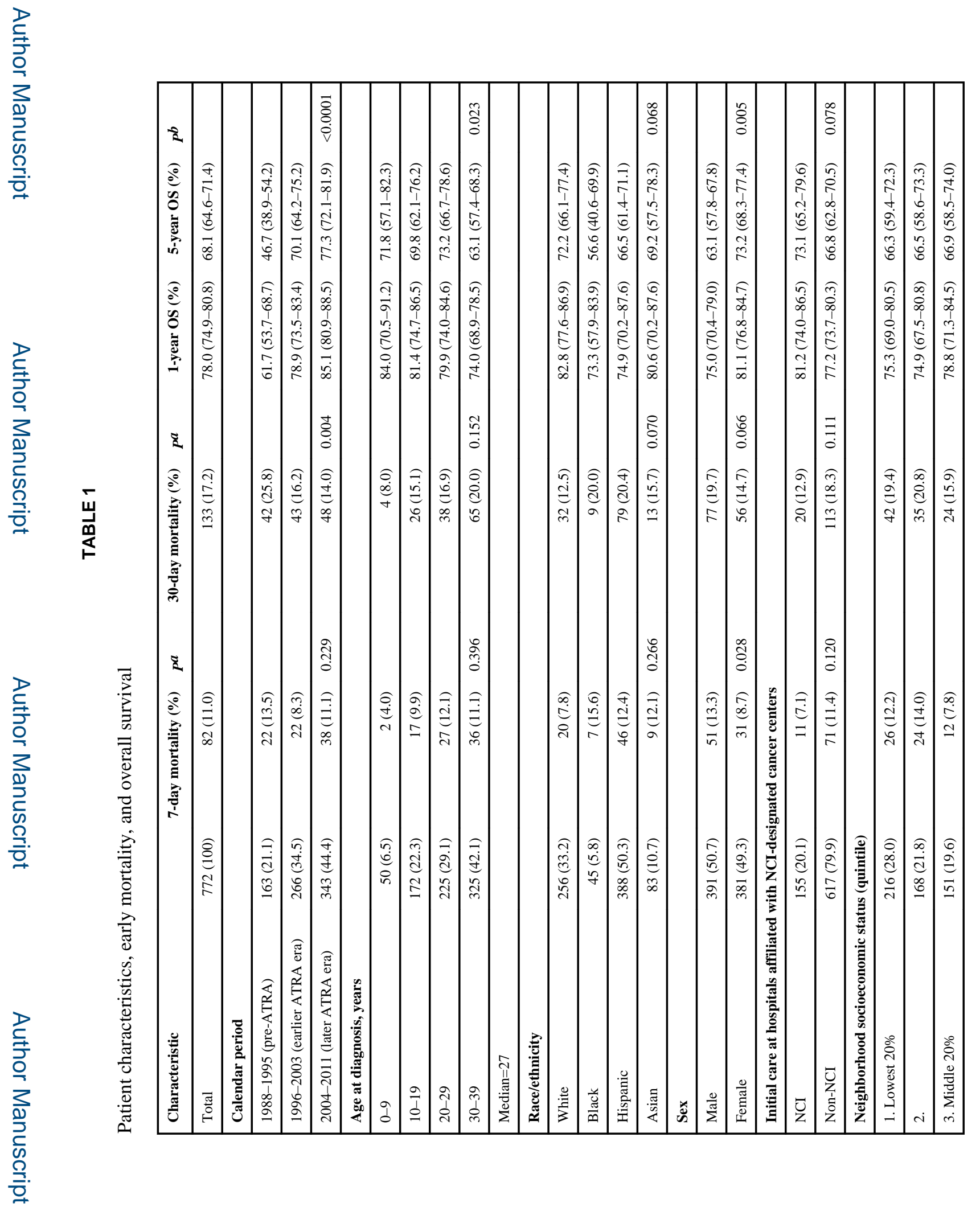

Cancer. Author manuscript; available in PMC 2016 November 15. 


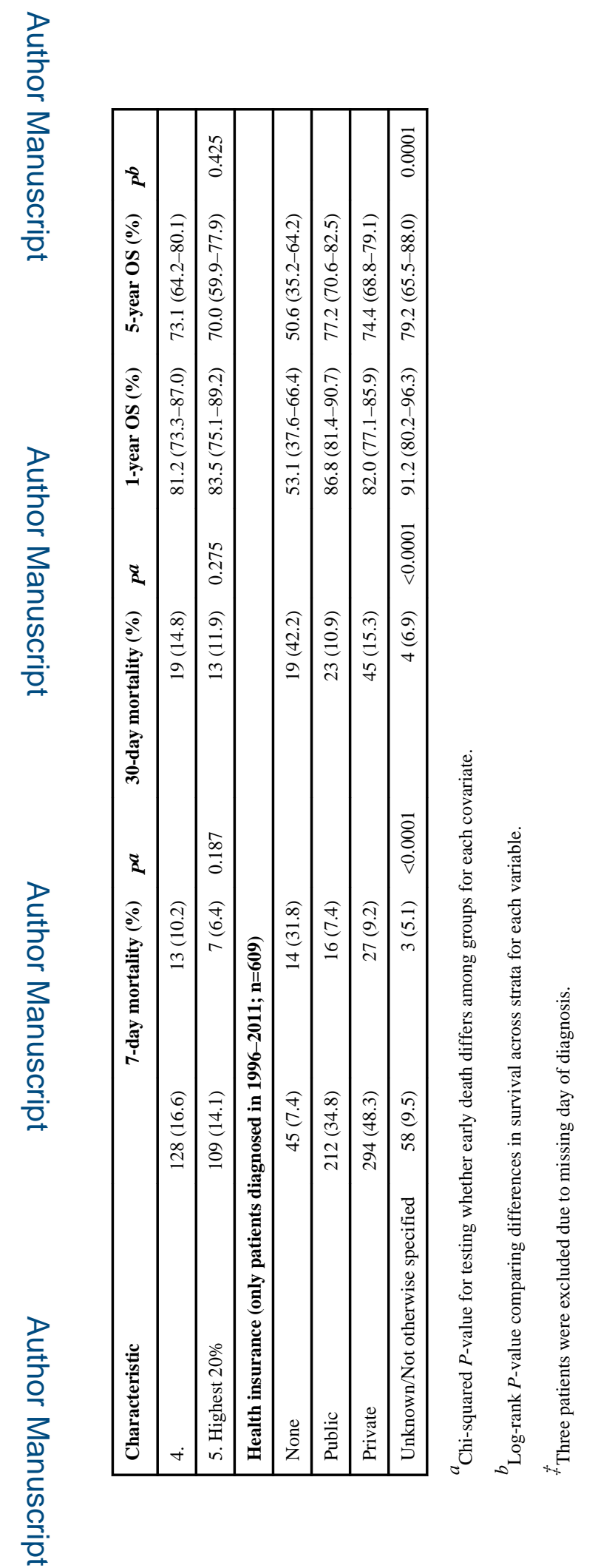




\section{TABLE 2}

Relation of sociodemographic and clinical factors to 30-day mortality

\begin{tabular}{|c|c|c|c|c|}
\hline \multirow[b]{2}{*}{ Factor } & \multicolumn{4}{|c|}{ Odds ratio for 30-day mortality } \\
\hline & $\begin{array}{r}\text { Unadjusted OR1 } \\
(95 \% \text { CI }) \\
(1988-2011)\end{array}$ & $\begin{array}{r}\text { Adjusted OR2 } \\
(95 \% \text { CI) } \\
(1988-2011)\end{array}$ & $\begin{array}{r}\text { Adjusted OR3 } \\
(95 \% \mathrm{CI}) \\
(1996-2011)\end{array}$ & $\begin{array}{r}\text { Adjusted OR4 } \\
(95 \% \text { CI }) \\
(1996-2011)\end{array}$ \\
\hline \multicolumn{5}{|l|}{ Calendar period } \\
\hline 1988-1995 (pre-ATRA) & $2.18(1.37-3.46)$ & $3.01(1.66-5.46)$ & N/A & N/A \\
\hline 1996-2003 (earlier ATRA era) & $1.20(0.77-1.87)$ & $1.39(0.80-2.43)$ & $1.41(0.81-2.46)$ & $1.30(0.74-2.30)$ \\
\hline 2004-2011 (later ATRA era) & 1 (base) & 1 (base) & 1 (base) & 1 (base) \\
\hline \multicolumn{5}{|l|}{ Sex } \\
\hline Male & $1.42(0.97-2.07)$ & $1.21(0.76-1.96)$ & $1.22(0.70-2.13)$ & $1.18(0.67-2.08)$ \\
\hline Female & 1 (base) & 1 (base) & 1 (base) & 1 (base) \\
\hline \multicolumn{5}{|l|}{ Age at diagnosis (years) } \\
\hline $0-9$ & 1 (base) & 1 (base) & 1 (base) & 1 (base) \\
\hline $10-19$ & $2.06(0.69-6.22)$ & $1.90(0.54-6.74)$ & $1.78(0.40-7.95)$ & $2.01(0.44-9.18)$ \\
\hline $20-29$ & $2.36(0.80-6.95)$ & $1.83(0.52-6.42)$ & $1.67(0.38-7.38)$ & $1.72(0.38-7.78)$ \\
\hline $30-39$ & $2.90(1.01-8.35)$ & $2.48(0.73-8.45)$ & $2.61(0.61-11.1)$ & $2.61(0.60-11.4)$ \\
\hline \multicolumn{5}{|l|}{ Race/ethnicity } \\
\hline White & 1 (base) & 1 (base) & 1 (base) & 1 (base) \\
\hline Black & $1.75(0.77-3.97)$ & $1.82(0.63-5.20)$ & $2.48(0.72-8.51)$ & $2.37(0.68-8.31)$ \\
\hline Hispanic & $1.79(1.14-2.79)$ & $2.13(1.16-3.89)$ & $2.20(1.04-4.63)$ & $2.23(1.01-4.92)$ \\
\hline Asian & $1.3(0.65-2.61)$ & $1.35(0.56-3.26)$ & $1.11(0.36-3.51)$ & $1.24(0.39-3.87)$ \\
\hline \multicolumn{5}{|c|}{ Neighborhood socioeconomic status (quintiles) } \\
\hline 1. Lowest $20 \%$ & $1.80(0.92-3.52)$ & $1.03(0.44-2.44)$ & $0 / 83((0.28-2.52)$ & $0.87(0.27-2.80)$ \\
\hline 2. & $1.91(0.96-3.79)$ & $1.08(0.46-2.53)$ & $0.99(0.33-2.92)$ & $1.03(0.33-3.20)$ \\
\hline 3. Middle $20 \%$ & $1.38(0.67-2.84)$ & $0.93(0.39-2.23)$ & $0.88(0.29-2.72)$ & $0.93(0.29-3.01)$ \\
\hline 4. & $1.30(0.61-2.77)$ & $0.81(0.32-2.02)$ & $0.79(0.25-2.53)$ & $0.83(0.25-2.72)$ \\
\hline 5. Highest $20 \%$ & 1 (base) & 1 (base) & 1 (base) & 1 (base) \\
\hline \multicolumn{5}{|c|}{ Initial care at hospitals affiliated with NCI-designated cancer centers } \\
\hline Yes & 1 (base) & 1 (base) & 1 (base) & 1 (base) \\
\hline No & $1.53(0.92-2.55)$ & $1.07(0.57-2.00)$ & $1.30(0.62-2.72)$ & $1.19(0.55-2.56)$ \\
\hline \multicolumn{5}{|c|}{ Health insurance (limited to patients diagnosed in 1996-2011; n=609) } \\
\hline None & $3.91(2.01-7.62)$ & N/A & N/A & $2.67(1.10-6.52)$ \\
\hline Public & $0.66(0.39-1.13)$ & N/A & N/A & $0.66(0.32-1.33)$ \\
\hline Private & 1 (base) & N/A & N/A & 1 (base) \\
\hline Unknown/NOS & $0.40(0.14-1.17)$ & N/A & $\mathrm{N} / \mathrm{A}$ & $0.22(0.06-0.79)$ \\
\hline
\end{tabular}

Abbreviations: OR, odds ratio; CI, confidence interval; NCI, National Cancer Institute.

All multivariable comparisons were adjusted for chemotherapy $(\mathrm{Y} / \mathrm{N})$ and all variables in the table unless otherwise noted.

OR1: unadjusted model (1988-2011), OR2: adjusted model without insurance (1988-2011), OR3: adjusted model without insurance (1996-2011), OR4: adjusted model with insurance (1996-2011). 


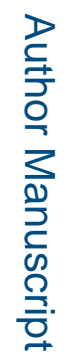

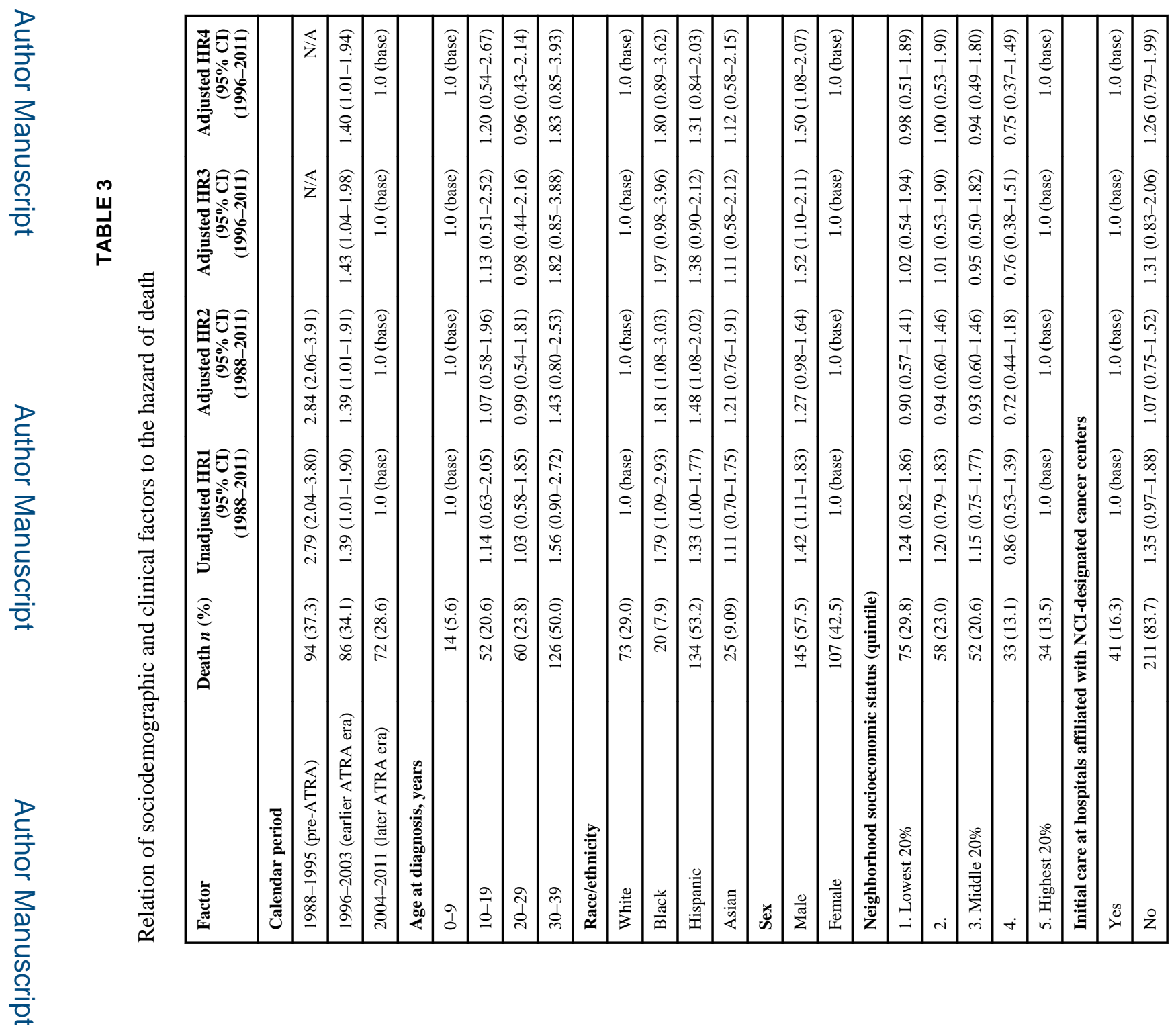

Cancer. Author manuscript; available in PMC 2016 November 15. 
